\begin{tabular}{|c|l|}
\hline Title & Electrical properties and applications of carbon nanotube composites \\
\hline Author(s) & Sano, Eiichi; A kiba, Eiji \\
\hline Citation & $\begin{array}{l}\text { International journal of nanotechnology, 13(7), 524-532 } \\
\text { https://doi.org/L0.1504/JNT.2016.078544 }\end{array}$ \\
\hline Issue Date & 2016-07 \\
\hline Doc URL & http://hdl.handle.net/2115/64552 \\
\hline Type & article(author version) \\
\hline File Information & Template Int J Nanotechnol_Sano.pdf \\
\hline
\end{tabular}

Instructions for use 


\title{
Electrical properties and applications of carbon nanotube composites
}

\author{
Eiichi Sano * \\ Research Center for Integrated Quantum Electronics \\ Hokkaido University \\ Sapporo 060-8628, Japan \\ Fax: +81-11-716-6004 E-mail: esano@rciqe.hokudai.ac.jp \\ ${ }^{*}$ Corresponding author
}

\section{Eiji Akiba}

Research \& Development Department Department

Kuraray Living Co. Ltd.

Osaka 530-8611, Japan

Fax: + E-mail: EIJI_AKIBA@kuraray.co.jp

\begin{abstract}
Many types of electromagnetic (EM) absorbers have been developed to convert EM energy into heat and to absorb EM wave. However, multilayer EM absorbers are complicated to design, fabricate, and dispose of. In this work, we present a simple and scalable design method to achieve a single-layer electromagnetic absorber with a high absorption coefficient in a wide range of frequency bands. On the basis of the design, we develop two types of nonwoven fabrics with high porosity in which the fibers are coated with multiwalled carbon nanotubes (MWCNTs). The absorption coefficients exceed 0.9 for the 5-mm-thick MWCNT-coated nonwoven fabric measured in the 50-67 $\mathrm{GHz}$ rang. An absorption coefficient of 0.94 at $5.8 \mathrm{GHz}$ is expected to be obtained by using 50-mm MWCNT-coated fabric with a lower MWCNT content. The present MWCNT-coated nonwoven fabrics will be useful materials for high performance EM absorbers.
\end{abstract}

Keywords: Carbon nanotube, multi-walled carbon nanotube, nonwoven fabric, composite, conductivity, permittivity, electromagnetic absorber, electromagnetic interference problems

Reference for publisher use only

Biographical notes: Eiichi Sano received the B. S., M. S., and Ph. D degrees from the University of Tokyo, Tokyo, Japan, in 1975, 1977, and 1998, respectively. From 1977 to 2001, he was with NTT laboratories, where he worked on MOS device physics, mixed analog/digital MOS ULSIs, ultrafast MSM photodetectors, electrooptic sampling, high-speed electronic and optoelectronic ICs. In 2001, he joined the Research Center for Integrated Quantum Electronics, Hokkaido University, Japan, as a Professor. His current research interests include nanocarbon materials, high-speed devices, and lowpower CMOS circuits. He has published more than 250 papers in major journals and conference proceedings related to these research areas. Prof. Sano is a member of the IEEE and JSAP and a Fellow of IEICE.

Eiji Akiba received the B.S. and M.S. from the Kyoto Institute of Technology, Kyoto, Japan, in 1982 and 1984 respectively. From 1984 to 1990, he worked on 


\begin{abstract}
Tamashima-plant of KURARAY in charge of polyester staple fiber production. From 1990 to 1998, he worked on Saijo-plant of KURARAY in charge of polyester filament development. From 1998 to 2005, he worked on the headquarters of KURARAY in charge of planning and development of apparel materials. In 2005, he joined KURARAY LIVING as a R\&D manager. His current work is commercialization of "CNTEC" (carbon nanotubes coated electro-conductive yarn), and "SROPE" (high content functional chemical agent masterbatch).
\end{abstract}

\title{
1 Introduction
}

Carbon nanotubes (CNTs) have been attracting much attention due to their excellent electrical, mechanical, and thermal properties [1]. Inclusion of CNTs into base materials improves the performance of the materials and even produces additional functions for them. The extremely high conductivity and large aspect ratio of CNTs are very effective for filler in conductive composite materials. The application areas of these materials include transparent conductive sheets, battery electrodes, and electromagnetic (EM) absorbers. As wireless equipment has become more convenient, electromagnetic interference (EMI) problems have become more serious. Many types of EM absorbers have been developed to convert EM energy into heat and to absorb EM wave. In the design of commonly used multilayer EM absorbers with metal backplanes, the thickness, permittivity, and/or conductivity of each layer must be adjusted to achieve high absorbance at a target frequency [2]. CNT composites are effective to achieve wide ranges of permittivity and conductivity [3-9] and to widen design flexibility of EM absorbers. A high conductivity of more than $10^{3} \mathrm{~S} / \mathrm{m}$ has been reported for both singlewalled (SW) [10] and multi-walled (MW) [11] CNT composites, while a large real permittivity of 200 at $18 \mathrm{GHz}$ has been observed for MWCNT/cellulose composite paper [12]. However, multilayer EM absorbers are complicated to design, fabricate, and dispose of. Therefore, the purpose of this work is to present CNT composites with the complex permittivity required to achieve a single-layer absorber with a high absorption coefficient.

\section{Experimental details}

\subsection{Material design}

The coefficients of transmission $T$, reflection $R$, and absorption $A$ for a single-layer conductive material were calculated by taking into account the multiple reflections at both surfaces of the material [13] (see Appendix A). The permeability of the material was assumed to be equal to that of vacuum, while the complex permittivity in the material was expressed as

$$
\varepsilon=\varepsilon_{0}\left(\varepsilon_{r}^{\prime}-j \varepsilon_{r}^{\prime \prime}\right)=\varepsilon_{0}\left(\varepsilon_{r}^{\prime}-j \frac{\sigma}{\omega \varepsilon_{0}}\right)
$$

Here, $\varepsilon_{0}$ is the permittivity of vacuum, $\sigma$ is the conductivity, and $\omega$ is the angular frequency. Figure 1(a) shows a contour plot of the calculated absorption coefficient in the $\varepsilon_{\mathrm{r}}$ - $\sigma$ plane for the normal incident to the material (thickness $d=5 \mathrm{~mm}$ ) at $60 \mathrm{GHz}$. The 
calculation result indicated that an absorption coefficient of 0.95 can be achieved with only a single layer when $\varepsilon_{r}$ ' is close to that of the free space and $\sigma$ is around $3 \mathrm{~S} / \mathrm{m}$. This design method is simple and scalable. When used in $5.8 \mathrm{GHz}$ ISM (Industry-ScienceMedical) band (e.g. electronic toll collection systems in Japan), the thickness $d$ should be increased to $\sim 50 \mathrm{~mm}$ and the conductivity $\sigma$ should be decreased to $0.3 \mathrm{~S} / \mathrm{m}$ (keeping $\varepsilon_{r}$ " constant). This is clearly shown in Figure 1(b). In Figure 1(a) and (b), the values of $\varepsilon_{r}$ ' and $\sigma$ reported for the CNT composites [3-9] are plotted, although they were not measured at 5.8 or $60 \mathrm{GHz}$. The reported CNT composites seemed to be unsuitable for the single-layer absorbers with sufficient absorption coefficients at both frequencies. It is difficult to obtain CNT composites with $\varepsilon_{r}$ ' close to 1.0 using dense base materials such as silica, ceramic, and even polymer, because their dielectric constants are larger than 2 . CNT composites using porous materials (e.g. nonwoven fabrics with high porosity) as base materials seem to very effectively achieve the $\varepsilon_{r}$ ' and $\sigma$ required to achieve high absorption coefficients [13].

Figure 1 Contour plots of calculated absorption coefficient in $\varepsilon_{r}$ '- $\sigma$ plane for single-layer conductive materials with thicknesses of (a) $5 \mathrm{~mm}$ at $60 \mathrm{GHz}$ and (b) $50 \mathrm{~mm}$ at $5.8 \mathrm{GHz}$. Open circles: reported values of $\varepsilon_{r}$ ' and $\sigma$ for CNT composites measured at $1 \mathrm{GHz}$ [3], $12 \mathrm{GHz}[6], 18 \mathrm{GHz}[5,9]$, and $40 \mathrm{GHz}[4,7,8]$

(a)

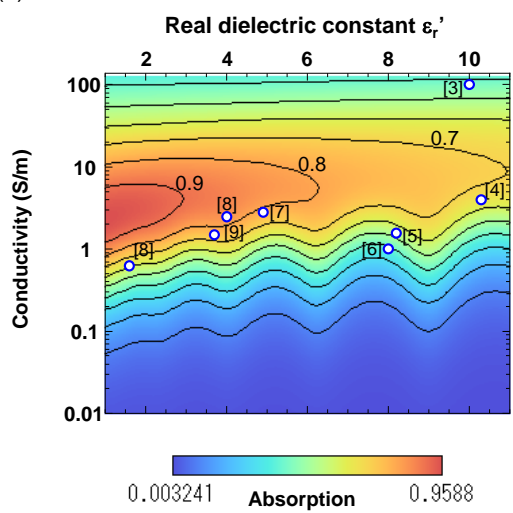

(b)

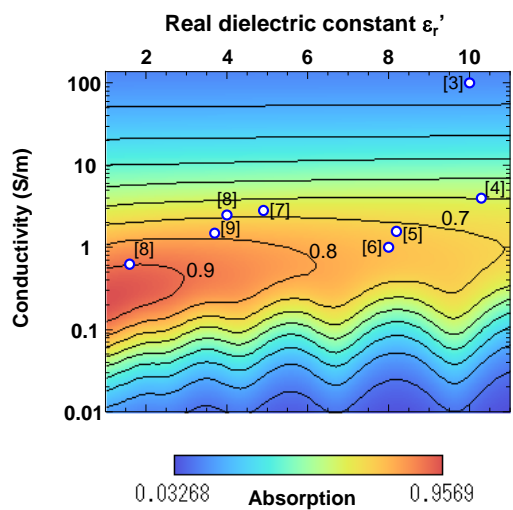




\subsection{Fabrication and evaluation methods}

Two types of MWCNT-coated nonwoven fabrics were fabricated: one (fabric I) for 60 $\mathrm{GHz}$ use and the other (fabric II) for $5.8 \mathrm{GHz}$ use. The MWCNTs used for fabrics I and II were produced by Nanocyl S.A. and CNano Technology Ltd., respectively. The basic fabric was polyester thermally bonded nonwoven fabric (Nishikawa Rose, specific gravity $=0.093 \mathrm{~g} / \mathrm{cm}^{3}$ ). A sheet of nonwoven fabric was dipped into the MWCNT-water suspension, wrung by a mangle machine, and dried in a hot-air oven at $90{ }^{\circ} \mathrm{C}$ to obtain MWCNT-coated nonwoven fabric [13]. The MWCNT contents of fabrics I and II were respectively 2.4 and $0.6 \mathrm{wt} \%$. The porosity of both MWCNT-coated fabrics was $95 \%$.

The fabricated absorbers were evaluated using a vector network analyzer (Agilent, E8361C) and materials measurement software (Agilent, 85071E) on the basis of a free space method (see the inset of Figure 2). Two horn antennas (Schwarzbeck, BBHA9120C) were used in 3-18 GHz, while two standard gain horn antennas (Millitech, SGH-15) were used in 50-67 GHz. The distances between the two antennas were 16 and $20 \mathrm{~cm}$ for 3-18 GHz and 50-67 GHz measurements, respectively. The two-port system was calibrated by using the gated reflect line calibration method. All the absorbers were $21 \times 21 \mathrm{~cm}$. In this measurement setup, the absorber was large enough to avoid any environment effects. The absorption coefficients were calculated as $1-\left|S_{11}\right|^{2}-\left|S_{21}\right|^{2}$ from measured scattering parameters $\left(S_{11}\right.$ and $\left.S_{21}\right)$. The complex permittivity values of the absorbers were extracted from the measured scattering parameters by using the materials measurement software. The accuracy of the free space measurement system had been confirmed by comparing the measured results with those measured with a waveguide method in the 50-67 GHz band [13]. See the website of Keysight Technologies [14] for more details about the measurement method.

\section{Results and discussion}

Figure 2 shows the measured absorption coefficient of fabric I with a thickness of $5 \mathrm{~mm}$. High absorption coefficients larger than 0.9 were achieved in the $50-67 \mathrm{GHz}$ frequency range. The dashed line in Figure 2 was calculated by Eqs. (A1)-(A4), where the complex permittivity of fabric I was approximated by the Maxwell-Garnett and Drude-Lorentz models (see Appendix B). Good agreement between measured and calculated absorption coefficients was obtained in the 50-67 GHz frequency range. In contrast, the calculations deviated from the measurements in the $3-18 \mathrm{GHz}$ frequency range. To investigate the reason for the discrepancy in the $3-18 \mathrm{GHz}$ frequency range, we examined the permittivity of fabric I extracted from measured scattering parameters by using the materials measurement software. Figure 3 compares measured and calculated relative permittivity (real part $\varepsilon_{r}^{\prime}$ and imaginary part $\varepsilon_{r}$ ”). The calculations using the MaxwellGarnett (M-G) model closely matched the measured permittivity in the $50-67 \mathrm{GHz}$ frequency range. Although the $M-G$ model fairly reproduced the measured $\varepsilon_{r}$, it failed to reproduce $\varepsilon_{r}$ " in the 3-18 GHz frequency range. The substitution of Eq. (B5) into Eq. (B1) reveals that $\varepsilon_{r}$ ' and $\varepsilon_{r}$ " approach $\varepsilon_{i}\{N+f(1-N)\} / N /(1-f)$ and 0 , respectively, at $0 \mathrm{~Hz}$. Since $\varepsilon_{r}$ " approaches 0 when the frequency diverges to infinity, $\varepsilon_{r}$ ” is convex upward as shown in Figure 3. The decreased $\varepsilon_{r}$ " in the M-G model was the major reason for the difference between measured and calculated absorption coefficients in the $3-18 \mathrm{GHz}$ frequency range.

Then we examined the often used Birchak model (see Appendix B). The dotted lines in Figure 3 were calculated by using the Birchak model. Although the Birchak model monotonically decreases both $\varepsilon_{r}$ ' and $\varepsilon_{r}$ " as the frequency increases, the deviation from the measured values were large. The dashed-dotted lines for $\varepsilon_{r}$ ” in Figure 3 were 
calculated by Eq. (1) with two conductivity values. Development of an appropriate model describing the permittivity at lower frequency region remains for future work.

Figure 2 Measured and calculated absorption coefficients of fabric I. Inset shows the measurement setup.

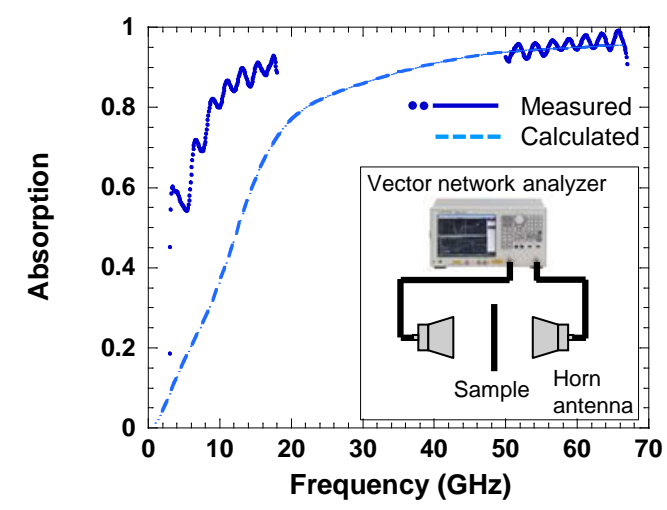

Figure 3 Measured and calculated relative permittivity of fabric I. Solid lines: measured. Dashed lines: calculated using Maxwell-Garnett (M-G) model. Dotted lines: calculated using Birchak model. Dashed-dotted lines: calculated by $\sigma /\left(\omega \varepsilon_{0}\right)$ with $\sigma=$ 2.1 and $1.3 \mathrm{~S} / \mathrm{m}$

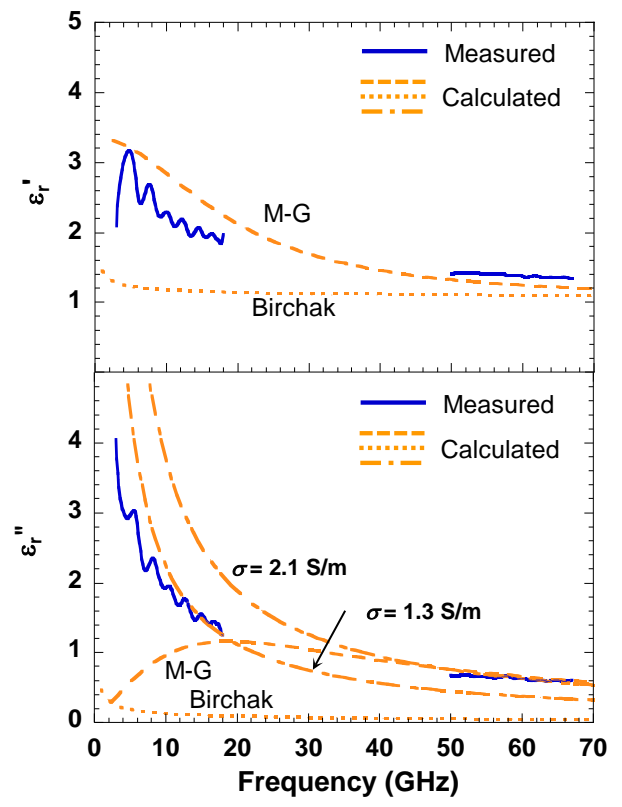

Figure 4 shows the dependences of absorption, transmission, and reflection coefficients on thickness for fabric II measured at $5.8 \mathrm{GHz}$. The solid lines were calculated using the measured permittivity of $\varepsilon_{r}{ }^{\prime}=1.42$ and $\varepsilon_{r}{ }^{\prime \prime}=0.69$. A good agreement 
was obtained between measured and calculated coefficients. The AC conductivity ( $\sigma=\omega \varepsilon_{r}$ " $\varepsilon_{0}$ ) was $0.22 \mathrm{~S} / \mathrm{m}$. The achieved $\varepsilon_{r}$ ' and $\sigma$ were close to the optimum values shown in Figure 1(b). An absorption coefficient of 0.94 was expected to be obtained by using 50-mm MWCNT-coated fabric.describing the permittivity at lower frequency region remains for future work.

Figure 4 Dependences of absorption, transmission, and reflection coefficients on thickness for fabric II at $5.8 \mathrm{GHz}$. Symbols: measured. Lines: calculated.

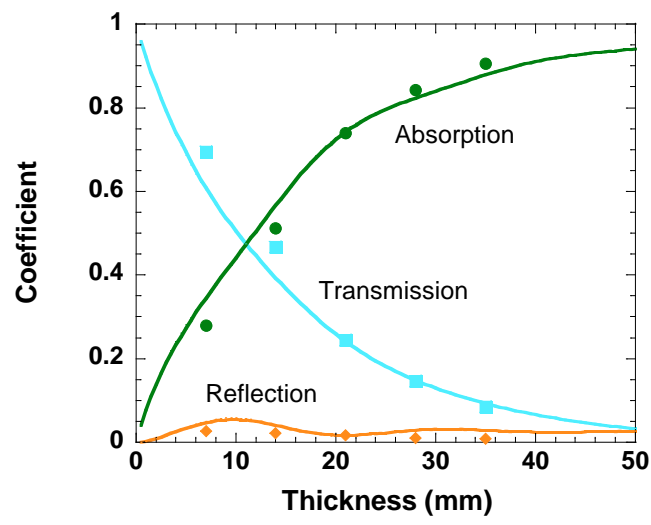

\section{Conclusions}

In this work, we presented a simple and scalable design method to achieve a single-layer electromagnetic absorber with a high absorption coefficient in a wide range of frequency bands. On the basis of the design, we developed two types of nonwoven fabrics with high porosity in which the fibers were coated with MWCNTs. The absorption coefficients exceeded 0.9 for the 5-mm-thick MWCNT-coated nonwoven fabric measured in the 50$67 \mathrm{GHz}$ range, which was in agreement with the calculations. An absorption coefficient of 0.94 at $5.8 \mathrm{GHz}$ was expected to be obtained by using 50-mm MWCNT-coated fabric with a lower CNT content. The MWCNT-coated nonwoven fabrics opened up a new region in the $\varepsilon_{r}$ '- $\sigma$ plane. The present MWCNT-coated nonwoven fabrics will be useful materials for high performance electromagnetic absorbers.

\section{Appendix A}

The coefficients of transmission $T$, reflection $R$, and absorption $A$ for a three-layer system are given by

$$
T=t t^{*}, R=r r^{*}, A=1-T-R,
$$

where

$$
t=\frac{t_{12} t_{23} \exp (-j \Delta)}{1+r_{12} r_{23} \exp (-2 j \Delta)}
$$

and

$$
r=\frac{r_{12}+r_{23} \exp (-2 j \Delta)}{1+r_{12} r_{23} \exp (-2 j \Delta)}
$$

with 


$$
\Delta=k_{0} n_{2} d \cos \theta_{r} .
$$

Here, $t^{*}$ and $r^{*}$ are respectively the complex conjugates of $t$ and $r, r_{m n}$ and $t_{m n}$ are respectively the reflection and transmission ratios of the complex electric field amplitudes (from medium $m$ to $n$ ), $k_{0}$ is the wave number in the free space, $n_{2}$ and $d$ are respectively the complex index of refraction and the thickness of Medium 2, $\theta_{r}$ is the refraction angle calculated by Snell's law, and $j=\sqrt{-1}$. The ratios $r_{m n}$ and $t_{m n}$ for perpendicular polarization are given by

$$
r_{m n}=\frac{\eta_{n} \cos \theta_{i}-\eta_{m} \cos \theta_{r}}{\eta_{n} \cos \theta_{i}+\eta_{m} \cos \theta_{r}} \text { and } t_{m n}=r_{m n}+1
$$

with the wave impedances $\eta_{m}$ and $\eta_{n}$ of Medium $m$ and $n$ [15]. A single-layer absorber was examined by treating Media 1 and 3 as air.

\section{Appendix B}

According to the Maxwell-Garnett model, the effective permittivity of CNT composites is given by [16]

$$
\varepsilon_{\text {eff }}=\varepsilon_{i} \frac{[N+f(1-N)] \varepsilon_{C N T}+(1-N)(1-f) \varepsilon_{i}}{N(1-f) \varepsilon_{C N T}+(f N+1-N) \varepsilon_{i}} .
$$

Here, $\varepsilon_{i}$ is the dielectric constant of a base material, $f$ is the filling factor (CNT content), and $N$ is the geometrical factor expressed as

$$
N \approx \frac{r^{2}}{a^{2}} \ln \frac{a}{r}
$$

for CNTs with a length of $2 a$ and a radius of $r$. The dielectric constant $\varepsilon_{i}$ of a porous base material with a porosity of $p$ is expressed as

$$
\varepsilon_{i}=\varepsilon_{\text {air }} p+\varepsilon_{d}(1-p) \text {, }
$$

where $\varepsilon_{d}$ is the dielectric constant of solid base material, and $\varepsilon_{a i r}=1$. The filling factor $f$ in Eq. (B1) is approximated by

$$
f=(1-p) v
$$

where $v$ is the volume fraction of CNTs. The dielectric constant of CNTs was approximated by the Drude-Lorentz model as in the work of Jeon et al. [16]

$$
\varepsilon_{C N T}=\varepsilon^{\infty}-\frac{\omega_{p}^{2}}{\omega^{2}-j \Gamma \omega}+\frac{\omega_{p l}^{2}}{\omega_{l}^{2}-\omega^{2}+j \Gamma_{l} \omega} .
$$

The parameter values deduced from terahertz time-domain spectroscopy measurements for SWCNTs [16] were used in the calculation. They were $\varepsilon^{\infty}=3.24$, $\omega_{p} / 2 \pi=5.4 \mathrm{THz}, \Gamma / 2 \pi=1.17 \mathrm{THz}, \omega_{p l} / 2 \pi=6.19 \mathrm{THz}, \omega_{l} / 2 \pi=2.4 \mathrm{THz}$, and $\Gamma_{l} / 2 \pi=4.56$ THz. Although they were deduced for SWCNTs, these parameter values well reproduced the measured permittivity of fabric I in the 50-67 GHz frequency region.

The Birchak model gives the effective permittivity of CNT composites as

$$
\varepsilon_{\text {eff }}^{\beta}=f \varepsilon_{C N T}^{\beta}+(1-f) \varepsilon_{i}^{\beta}
$$
with $\beta=1 / 2$ [17].

\section{Acknowledgements}

The present study was partly supported by a Grant-in-Aid for Scientific Research from the Ministry of Education, Culture, Sports, Science and Technology, Japan. 


\section{References}

1 Baughman R.H., Zakhidov A.A. and de Heer W.A. (2002) 'Carbon nanotubes-the route toward applications’, Science, Vol. 297, pp. 787-792.

2 Matous K. and Dvorak G.J. (2003) 'Optimization of electromagnetic absorption in laminated composite plates’, IEEE Trans. Mag., Vol. 39, pp. 1827-1835.

3 Konyushenko E.N., Kazantseva N.E., Stejskal J., Trchova M., Kovarova J., Sapurina I., Tomishko M.M., Demicheva O.V. and Prokes J. (2008) 'Ferromagnetic behavior of polyaniline-coated multi-wall carbon nanotubes containing nickel nanoparticles', J. Mag. Mag. Mater., Vol. 320, pp. 231-240.

4 Xiang C., Pan Y. and Guo J. (2007) 'Electromagnetic interference shielding effectiveness of multiwalled carbon nanotube reinforced fused silica composites’, Ceramics International, Vol. 33, pp. 1293-1297.

5 Zhu H., Lin H., Guo H. and Yu L. (2007) 'Microwave absorbing property of Fe-filled carbon nanotubes synthesized by a practical route’, Mater. Sci. Eng. B, Vol. 138, pp. 101-104.

6 Xiang C., Pan Y., Liu X., Sun X., Shi X. and Guo J. (2005) 'Microwave attenuation of multiwalled carbon nanotube-fused silica composites’, Appl. Phys. Lett., Vol. 87, pp. 123103$1-3$.

7 Saib A., Bednarz L., Daussin R., Bailly C., Lou X., Thomassin J.M., Pagnoulle C., Detrembleur C., Jérôme R. and Huynen I. (2006) 'Carbon nanotube composites for broadband microwave absorbing materials’, IEEE Trans. Microwave Theory Tech., Vol. 54, pp. 27452754.

8 Thomassin J.M., Pagnoulle C., Bednarz L., Huynen I., Jerome R. and Detrembleur C. (2008) 'Foams of polycaprolactone/MWNT nanocomposites for efficient EMI reduction', J. Mater. Chem., Vol. 18, pp. 792-796.

9 Wu J. and Kong L. (2004) 'High microwave permittivity of multiwalled carbon nanotube composites', Appl. Phys. Lett., Vol. 84, pp. 4956-4958.

10 Aarab H., Baitoul M., Wery J., Almairac R., Lefrant S., Faulques E., Duvail J.L. and Hamedoun M. (2005) 'Electrical and optical properties of PPV and single-walled carbon nanotubes composite films', Synth. Met., Vol. 155, pp. 63-67.

11 Kim H.M., Kim K., Lee C.Y. and Joo J. (2004) 'Electrical conductivity and electromagnetic interference shielding of multiwalled carbon nanotube composites containing Fe catalyst', Appl. Phys. Lett., Vol. 84, pp. 589-591.

12 Imai M., Akiyama K., Tanaka T. and Sano E. (2010) 'Highly strong and conductive carbon nanotube/cellulose composite paper’, Compos. Sci. Technol., Vol. 70, pp. 1564-1570.

13 Sano E. and Akiba E. (2014) 'Electromagnetic absorbing materials using nonwoven fabrics coated with multi-walled carbon nanotubes', Carbon, Vol. 78, pp. 463-468.

14 http://www.keysight.com/upload/cmc_upload/All/FreeSpaceSeminarRev2.pdf

15 Pozar D.M. (1998) Microwave engineering, $2^{\text {nd }}$ ed., John Wiley \& Sons, Inc., NJ.

16 Jeon T.I., Kim K.J., Kang C., Maeng I.H., Son J.H., An K.H., Lee J.Y. and Lee Y.H. (2004) 'Optical and electrical properties of preferentially anisotropic single-walled carbon-nanotube films in terahertz region', J. Appl. Phys., Vol. 95, pp. 5736-5740.

17 Karkkainen K.K., Sihvola A.H. and Nikoskinen K.I. (2000) 'Effective permittivity of mixtures: Numerical validation by the FDTD method,' IEEE Trans. Geoscience Remote Sensing, Vol. 38, pp. 1303-1308. 